\title{
Efficacy and safety of TE/TEC/intensive paclitaxel neoadjuvant chemotherapy for the treatment of breast cancer
}

\author{
YANG LIU ${ }^{1}$, ZHAOGUO XU ${ }^{2}$, ZHENYONG ZHANG ${ }^{2}$, GUANGFU WEN $^{3}$, JIAXING SUN ${ }^{4}$ and FENG HAN ${ }^{5}$ \\ Departments of ${ }^{1}$ Surgery, ${ }^{2}$ Oncology, ${ }^{3}$ Pediatric Intensive Care Medicine, ${ }^{4}$ Ultrasonography and \\ ${ }^{5}$ Medical Administration, Shengjing Hospital of China Medical University, Shenyang, Liaoning 110000, P.R. China
}

Received June 11, 2018; Accepted October 24, 2018

DOI: 10.3892/ol.2018.9658

\begin{abstract}
Efficacy and safety of paclitaxel/docetaxel + epirubicin (TE), paclitaxel/docetaxel + epirubicin + cytoxan (TEC) and intensive paclitaxel (IP) neoadjuvant chemotherapy (NCT) were compared for the treatment of breast cancer. The clinical data of 326 patients with stage II-III unilateral primary breast cancer treated in Shengjing Hospital of China Medical University from January 2012 to April 2016 were retrospectively analyzed. All patients received NCT for 4 cycles, including 115 cases of TE group, 109 cases of TEC group, and 102 cases of paclitaxel weekly group. The clinical efficacy was evaluated and complete response $(\mathrm{CR})+$ partial response $(\mathrm{PR})$ indicated clinically effective. The pathological effect was evaluated and the grade III $+\mathrm{IV}+\mathrm{V}$ indicated pathologically effective. The rates of clinical efficacy and pathological CR (pCR) were compared, and the incidence of adverse reactions was also observed. The effects of different molecular typing on clinical efficacy and pCR were compared. Our results showed that the clinical effective rates in TE, TEC and IP groups were $80.9,89.0$ and $77.5 \%$, respectively, and there were no statistically significant differences $(\mathrm{P}=0.074)$. The $\mathrm{pCR}$ rates in the three groups were $9.57,8.26$ and $5.88 \%$, respectively, and the differences were not statistically significant $(\mathrm{P}=0.602)$. The incidence rate of neutropenia was statistically different among the three groups of patients $(\mathrm{P}<0.001)$, which was the highest in TEC group and the lowest in IP group. There were no statistically significant differences in the incidence rates of adverse reactions $(\mathrm{P}>0.05)$. Estrogen receptor (ER)-negative, progesterone receptor (PR)-negative and human epidermal growth factor receptor-2 (HER-2)-positive states were significantly correlated with the high clinical effective rate and high pCR rate $(\mathrm{P}<0.05)$. In conclusion, IP has the lowest incidence rate of neutropenia. Additionally, ER-negative, PR-negative
\end{abstract}

Correspondence to: Dr Feng Han, Department of Medical Administration, Shengjing Hospital of China Medical University, 36 Sanhao Street, Heping, Shenyang, Liaoning 110000, P.R. China E-mail: xvxmq406@163.com

Key words: breast cancer, neoadjuvant chemotherapy, efficacy, adverse reactions, paclitaxel, molecular typing and HER-2-positive states are significantly correlated with the high clinical effective rate and high $\mathrm{pCR}$ rate.

\section{Introduction}

Neoadjuvant chemotherapy (NCT) refers to the preoperative chemotherapy for operable patients, which is one of the important treatment means for primary breast cancer in the progressive stage. Preoperative chemotherapy can reduce the tumor staging, locally control the lesion and improve the resectable rate, which can also provide the in vivo drug susceptibility information to guide the subsequent medication, so it has been widely used in clinic $(1,2)$. However, there have been no unified standards yet for selecting the NCT regimen and frequency for breast cancer. How to choose the most appropriate NCT regimen for patients has always been a hot issue in the breast cancer treatment research. According to clinical practice, the pathological complete response (pCR) of neoadjuvant systemic chemotherapy (NST) regimen combining anthracycline with paclitaxel is superior to the simple anthracycline-based chemotherapy regimen in the treatment of breast cancer. The advantages of anthracycline and paclitaxel in NST of breast cancer become increasingly prominent, and improving the efficacy and safety of chemotherapy is the emphasis in research on NST of breast cancer $(3,4)$.

In this study, the efficacy and adverse reactions were compared among paclitaxel/docetaxel + epirubicin (TE) regimen, paclitaxel/docetaxel + epirubicin + cytoxan (TEC) regimen and IP in NCT for breast cancer, and the efficacy of different chemotherapy regimens in NCT and their correlation with molecular typing were investigated, hoping to provide a theoretical basis and references for the selection of NCT regimen and individualized treatment of breast cancer.

\section{Patients and methods}

Objects of study. A total of 326 patients diagnosed with breast cancer for the first time in Shengjing Hospital of China Medical University (Shenyang, China) from January 2012 to April 2016 were collected in this study. Breast cancer in all patients was confirmed pathologically via biopsy using hollow needle, and patients received no radiotherapy, chemotherapy or endocrine therapy before treatment. All patients had the evaluable unilateral primary tumor lesions, and received blood routine, liver 
Table I. Demographics and general clinical data of all breast cancer patients.

\begin{tabular}{|c|c|c|c|c|c|}
\hline Parameters & $\begin{array}{c}\text { TE group } \\
n=115\end{array}$ & $\begin{array}{c}\text { TEC group } \\
n=109\end{array}$ & $\begin{array}{l}\text { IP group } \\
n=102\end{array}$ & $\chi^{2}$ value & P-value \\
\hline Age (years) & & & & 0.507 & 0.776 \\
\hline$<50$ & $54(47.0 \%)$ & $52(47.7 \%)$ & $44(43.1 \%)$ & & \\
\hline$\geq 50$ & $61(53.0 \%)$ & $57(52.3 \%)$ & $58(56.9 \%)$ & & \\
\hline Menopausal status & & & & 0.029 & 0.986 \\
\hline Premenopausal & $52(45.2 \%)$ & $49(45.0 \%)$ & $47(46.1 \%)$ & & \\
\hline Postmenopausal & $63(54.8 \%)$ & $60(55.0 \%)$ & $55(53.9 \%)$ & & \\
\hline Clinical tumor size & & & & 1.802 & 0.772 \\
\hline $\mathrm{T}_{1}$ & $3(2.6 \%)$ & $2(1.8 \%)$ & $2(2.0 \%)$ & & \\
\hline $\mathrm{T}_{2}-\mathrm{T}_{3}$ & $87(75.7 \%)$ & $79(72.5 \%)$ & $70(68.6 \%)$ & & \\
\hline $\mathrm{T}_{4}$ & $25(21.7 \%)$ & $28(25.7 \%)$ & $30(29.4 \%)$ & & \\
\hline Lymph node metastasis & & & & 3.466 & 0.483 \\
\hline $\mathrm{N}_{\mathrm{O}}$ & $5(4.3 \%)$ & $3(2.8 \%)$ & $1(9.8 \%)$ & & \\
\hline $\mathrm{N}_{1}-\mathrm{N}_{2}$ & $73(63.5 \%)$ & $67(61.5 \%)$ & $71(69.7 \%)$ & & \\
\hline $\mathrm{N}_{3}$ & $37(32.2 \%)$ & $39(35.7 \%)$ & $30(29.5 \%)$ & & \\
\hline TNM stage & & & & 0.508 & 0.776 \\
\hline II & $81(70.4 \%)$ & $72(66.1 \%)$ & $69(67.6 \%)$ & & \\
\hline III & $34(29.6 \%)$ & $37(33.9 \%)$ & $33(32.4 \%)$ & & \\
\hline HER-2 status & & & & 0.295 & 0.863 \\
\hline+ & $56(48.7 \%)$ & $52(47.7 \%)$ & $46(45.1 \%)$ & & \\
\hline- & $59(51.3 \%)$ & $57(52.3 \%)$ & $56(54.9 \%)$ & & \\
\hline ER status & & & & 0.476 & 0.788 \\
\hline+ & $51(44.3 \%)$ & $51(46.8 \%)$ & $50(49.0 \%)$ & & \\
\hline- & $64(55.7 \%)$ & $58(53.2 \%)$ & $52(51.0 \%)$ & & \\
\hline PR status & & & & 0.435 & 0.805 \\
\hline+ & $47(40.9 \%)$ & $48(44.0 \%)$ & $46(45.1 \%)$ & & \\
\hline- & $68(59.1 \%)$ & $61(56.0 \%)$ & $56(54.9 \%)$ & & \\
\hline
\end{tabular}

TE, docetaxel and epirubicin; TEC, docetaxel, epirubicin and cyclophosphamide; IP, intensive paclitaxel; TNM, tumor-node-metastasis; HER-2, human epidermal growth factor receptor 2; ER, estrogen receptor; PR, progesterone receptor.

and kidney function, chest X-ray, abdominal ultrasound, bone scan, electrocardiography, echocardiography and pulmonary function examinations before treatment, and the patients with distant metastasis and severe cardiopulmonary dysfunction were excluded. Patients signed an informed consent, and this study was approved by the Medical Ethics Committee of Shengjing Hospital of China Medical University.

Patients were aged $46.4 \pm 7.9$ years, including 150 patients aged $<50$ years and 176 patients aged $\geq 50$ years. There were 148 premenopausal patients and 178 postmenopausal patients. According to the tumor-node-metastasis (TNM) staging of tumor developed by the American Joint Committee on Cancer (AJCC) and the Union for International Cancer Control (UICC), there were 7 patients in T1, 236 patients in T2-3 and 83 patients in T4. According to preoperative axillary lymph node ultrasound and puncture pathology, no axillary lymph node metastasis was defined as N0, axillary lymph node metastasis as N1, mixed type as N2, and axillary lymph node metastasis complicated with internal mammary lymph node metastasis or supraclavicular lymph node metastasis as N3, so there were 9 patients in N0, 211 patients in N1-2 and 106 patients in N3. Estrogen receptor (ER) was positive in 152 cases and negative in 174 cases. Progesterone receptor (PR) was positive in 142 cases and negative in 184 cases. Human epidermal growth factor receptor-2 (HER-2) was positive in 154 cases and negative in 172 cases. There were no statistically significant differences in age $(\mathrm{P}=0.776)$, menstrual status $(\mathrm{P}=0.986)$, tumor size $(\mathrm{P}=0.772)$, lymph node metastasis $(\mathrm{P}=0.483)$, clinical TNM staging $(\mathrm{P}=0.776)$ and molecular typing $(\mathrm{P}>0.05)$, among the patients enrolled, and data were comparable (Table I).

Patients and methods. TE regimen: $75 \mathrm{mg} / \mathrm{m}^{2}$ docetaxel $+75 \mathrm{mg} / \mathrm{m}^{2}$ epirubicin, intravenous drip at $1 \mathrm{st}$ day, and 21 days as 1 cycle. TEC regimen: $75 \mathrm{mg} / \mathrm{m}^{2}$ docetaxel + $75 \mathrm{mg} / \mathrm{m}^{2}$ epirubicin $+600 \mathrm{mg} / \mathrm{m}^{2}$ cytoxan, intravenous drip at 1 st day, and 21 days as 1 cycle. IP: weekly administration of $75 \mathrm{mg} / \mathrm{m}^{2}$ paclitaxel. The $3 \mathrm{kinds}$ of chemotherapy regimens were all performed for 4 cycles. At $0.5 \mathrm{~h}$ before administration of chemotherapeutic drugs, $10 \mathrm{mg}$ dexamethasone was 
Table II. Clinical effective rates in three neoadjuvant chemotherapy groups.

\begin{tabular}{lccccr}
\hline Parameters & $\begin{array}{c}\text { TE group } \\
\mathrm{n}=115\end{array}$ & $\begin{array}{c}\text { TEC group } \\
\mathrm{n}=109\end{array}$ & $\begin{array}{c}\text { IP group } \\
\mathrm{n}=102\end{array}$ & $\chi^{2}$ value & P-value \\
\hline CCR+CPR & 93 & 97 & 79 & 5.197 & 0.074 \\
SD+PD & 22 & 12 & 23 & & \\
Clinical effective rate & $80.9 \%$ & $89.0 \%$ & $77.5 \%$ & & \\
\hline
\end{tabular}

TE, docetaxel and epirubicin; TEC, docetaxel, epirubicin and cyclophosphamide; IP, intensive paclitaxel; CCR, clinical complete response; $\mathrm{CPR}$, clinical partial response; $\mathrm{SD}$, stable disease; $\mathrm{PD}$, progressive disease.

routinely given to prevent allergy, proton pump inhibitor (omeprazole or lansoprazole) to suppress acid, and 5-hydroxytryptamine inhibitor (tropisetron hydrochloride or ramosetron) to stop vomiting. When the absolute value of neutrophils was $<2 \times 10^{9} / 1$, the recombinant human granulocyte colony-stimulating factor (300 $\mu \mathrm{g}$ Ruibai or $150 \mu \mathrm{g}$ filgrastim) was injected subcutaneously to increase leukocytes. During chemotherapy, the liver function was monitored, and liver protecting treatment was performed if the level of alanine aminotransferase (ALT) or aspartate transaminase (AST) was 1.5 times higher than the normal high value. Patients received echocardiography again if the heart rate was significantly accelerated compared with that before chemotherapy or arrhythmia occurred in electrocardiogram. When the accumulated dose of anthracycline was $300 \mathrm{mg} / \mathrm{m}^{2}$, dexrazoxane was given to protect the heart. After each cycle of chemotherapy, B-ultrasound and clinical physical examinations were performed to evaluate clinical efficacy and adverse reactions. If the disease was progressive according to the evaluation, the chemotherapy regimen would be changed or operative treatment would be considered.

Pathological evaluation. After pathological diagnosis, immunohistochemical analysis was performed. ER, PR, HER-2 status and proliferation index (percentage of Ki-67-stained cells) in the paraffin-embedded tumor samples were detected via immunohistochemical method. The intensity and percentage of each antibody of the stained cells were detected. According to the regulations of the American Society of Clinical Oncology and College of American Pathologists (ASCO-CAP) in 2010, ER and PR were considered as negative $(<1 \%)$ or positive $(\geq 1 \%)$, indicating the percentage of nuclear immune remission. At least $30 \%$ cells in HER-2positive patients were strongly stained in the membrane, and more than $14 \%$ positive staining in the cell nucleus indicated slightly higher level of Ki-67. Primary rabbit monoclonal ER antibody (dilution, 1:100; cat. no. ab79413); rabbit monoclonal PR antibody (dilution, 1:100; cat. no. ab16661); mouse monoclonal HER-2 (also called ErbB2) antibody (dilution, 1:100; cat. no. ab16901) and rabbit monoclonal Ki-67 antibody (dilution, 1:100; cat. no. ab16667) were all purchased from Abcam (Cambridge, MA, USA).

Therapeutic evaluation criteria. The clinical and pathological response effects of NCT were evaluated according to the Response Evaluation Criteria In Solid Tumors (RECIST, Version 1.1). At the end of treatment, the response rate was evaluated in 326 patients with breast cancer. Clinical complete response (CCR): no residual breast and axillary lymph nodes, clinical partial response (CPR): decrease of tumor diameter by $30 \%$ or above, progressive disease (PD): increase of the maximum tumor diameter by $20 \%$ or occurrence of new diseases, and stable disease (SD): tumors not meeting the criteria of objective PR or PD. In this study, CR or PR (PR+CR) indicated clinically effective, while SD and PD (SD+PD) indicated clinically ineffective. pCR referred to no cancerous component or only in situ cancerous component found in the primary lesions and draining lymph nodes in surgical specimens, and no distant metastases in liver, lung, bone and brain.

The adverse and toxic side effects were evaluated according to the CETAE grading criteria (grade I-V) of the National Cancer Institute (NCI). Grades III-IV toxic and side effects were the major causes affecting chemotherapy, and grade $\mathrm{V}$ indicated death.

Statistical analysis. Statistical Product and Service Solutions (SPSS) 13.0 software (SPSS, Chicago, IL, USA) was used for statistical processing. Test indexes in this study were normally distributed according to the W-test. Continuous variables were presented as mean \pm standard deviation, and the variance was homogeneous according to the Levene test. Chi-square test was used for categorical data. Comparison between multiple groups was performed by using one-way ANOVA test followed by post hoc test (Least Significant Difference). $\mathrm{P}<0.05$ was considered to indicate a statistically significant difference.

\section{Results}

Clinical effective rates in TE, TEC and IP groups. The clinical effective rates in TE, TEC and IP groups were 80.9\% (93/115), 89.0\% (97/109) and 77.5\% (79/102), respectively, displaying no statistically significant differences among the three groups $(\mathrm{P}=0.074)$ (Table II).

$p C R$ rates in TE, TEC and IP groups. The $\mathrm{pCR}$ rates in TE, TEC and IP groups were 9.57\% (11/115), 8.26\% (9/100) and $5.88 \%$ (6/96), respectively, showing no statistically significant differences among the three groups $(\mathrm{P}=0.602)$ (Table III).

Adverse reactions. After chemotherapy, adverse reactions in different degrees, such as neutropenia, thrombocytopenia, decreased hemoglobin, gastrointestinal reactions, hepatic dysfunction, cardiotoxicity and alopecia, occurred in the 
Table III. pCR rates in three neoadjuvant chemotherapy groups.

\begin{tabular}{lccccc}
\hline Parameters & $\begin{array}{c}\text { TE group } \\
\mathrm{n}=115\end{array}$ & $\begin{array}{c}\text { TEC group } \\
\mathrm{n}=109\end{array}$ & $\begin{array}{c}\text { IP group } \\
\mathrm{n}=102\end{array}$ & $\chi^{2}$ value & P-value \\
\hline pCR (cases) & 11 & 9 & 6 & 1.017 & 0.602 \\
Not pCR (cases) & 104 & 100 & 96 & & \\
pCR rate (\%) & 9.57 & 8.26 & 5.88 & & \\
\hline
\end{tabular}

pCR, pathological complete response; TE, docetaxel and epirubicin; TEC, docetaxel, epirubicin and cyclophosphamide; IP, intensive paclitaxel.

Table IV. Comparison of adverse reaction in three neoadjuvant chemotherapy groups.

\begin{tabular}{lccccr}
\hline Parameters & $\begin{array}{c}\text { TE group } \\
\mathrm{n}=115\end{array}$ & $\begin{array}{c}\text { TEC group } \\
\mathrm{n}=109\end{array}$ & $\begin{array}{c}\text { IP group } \\
\mathrm{n}=102\end{array}$ & $\chi^{2}$ value & P-value \\
\hline Neutropenia & $26(22.6 \%)$ & $41(37.6 \%)$ & $7(6.9 \%)$ & 28.40 & 0.001 \\
Anemia & $14(12.2 \%)$ & $20(18.3 \%)$ & $8(7.8 \%)$ & 5.261 & 0.072 \\
Thrombocytopenia & $22(19.1 \%)$ & $25(22.9 \%)$ & $12(11.8 \%)$ & 4.564 & 0.102 \\
Gastrointestinal AR & $75(65.2 \%)$ & $77(70.6 \%)$ & $60(58.8 \%)$ & 3.239 & 0.198 \\
Liver function damage & $29(25.2 \%)$ & $31(28.4 \%)$ & $17(16.7 \%)$ & 4.300 & 0.117 \\
Cardiotoxicity & $50(43.5 \%)$ & $53(48.6 \%)$ & $40(39.2 \%)$ & 1.905 & 0.386 \\
Alopecia & $78(67.8 \%)$ & $79(72.5 \%)$ & $62(60.8 \%)$ & 3.301 & 0.192 \\
\hline
\end{tabular}

TE, docetaxel and epirubicin; TEC, docetaxel, epirubicin and cyclophosphamide; IP, intensive paclitaxel; AR, adverse reaction.

three groups of patients. Such gastrointestinal reactions as nausea and vomiting occurred in 75 cases $(65.2 \%, 75 / 115)$ in TE group, 77 cases $(70.6 \%, 77 / 109)$ in TEC group and 60 cases $(58.8 \%, 66 / 102)$ in IP group, and there were no statistically significant differences $\left(\chi^{2}=3.239, \mathrm{P}=0.198\right)$. No severe nausea and vomiting (grade III and IV) occurred in the three groups of patients. Neutropenia occurred in 26 cases $(22.6 \%$, $26 / 115)$ in TE group, 41 cases $(37.6 \%, 41 / 109)$ in TEC group and 7 cases $(6.9 \%, 7 / 102)$ in IP group, and differences were statistically significant $\left(\chi^{2}=28.4, \mathrm{P}<0.001\right)$. The pairwise comparisons revealed that the incidence rate of neutropenia in TEC group was significantly higher than that in TE group $(\mathrm{P}=0.0192)$, and it was also significantly higher in TE group than that in IP group $(\mathrm{P}=0.0012)$. There were 50 cases $(43.5 \%$, $50 / 115), 53$ cases $(48.6 \%, 53 / 109)$ and 40 cases $(39.2 \%$, $40 / 102)$ of cardiotoxicity in the three groups, respectively, and there were no statistically significant differences $\left(\chi^{2}=1.905\right.$, $\mathrm{P}=0.386)$. No cardiotoxicity above grade II occurred in the three groups. No statistically significant differences were found in the incidence of such adverse reactions as thrombocytopenia, decreased hemoglobin, hepatic dysfunction and alopecia among the three groups $(\mathrm{P}>0.05)$. All patients were treated with dexamethasone before chemotherapy, and there were no allergic reactions, such as bronchial asthma and hypotension, and pleural effusion. The adverse and toxic side effects were alleviated after treatment, and there was no case whose chemotherapy was terminated due to intolerance to adverse and toxic side effects of chemotherapy. Adverse and toxic side effects were compared among the three groups of patients (Table IV).
Impact of molecular typing on efficacy. The clinical effective rate under ER-negative state was $90.8 \%$, which was significantly higher than that under ER-positive state $(73.0 \%$, $\mathrm{P}<0.001)$. The clinical effective rate under PR-negative state was $92.9 \%$, which was obviously higher than that under PR-positive state $(69.0 \%, \mathrm{P}<0.001)$. The clinical effective rate under HER-2-positive state $(87.7 \%)$ was also remarkably higher than that under HER-2-negative state $(77.9 \%$, $\mathrm{P}=0.028$ ). The ER-negative, $\mathrm{PR}$-negative and HER-2-positive states were significantly correlated with the high $\mathrm{pCR}$ rate $(\mathrm{P}<0.05)($ Table V).

\section{Discussion}

Compared with postoperative adjuvant chemotherapy, the significance of NCT lies in lowering the tumor staging, transforming inoperable patients into operable patients, making it possible to perform breast-conserving surgery for all patients, providing in vivo drug susceptibility information to guide subsequent medications, reducing tumor cell viability to reduce intraoperative metastasis, eliminating small metastases in the body via early systemic therapy, and improving prognosis and providing prognostic indexes for patients with pCR (5). Currently, it is recognized internationally that NCT can be performed for patients who should receive postoperative chemotherapy. According to the recommendation of the National Comprehensive Cancer Network (NCCN) guideline, $\mathrm{NCT}$ can also be performed first for patients who can undergo local surgery of breast cancer in stage IIA, IIB and IIIA. Breast cancer patients with indications can benefit from NCT (6). 
Table V. Effect of molecular subtype on clinical effective rate and pCR in all breast cancer patients.

\begin{tabular}{lcccccr}
\hline $\begin{array}{l}\text { Parameter } \\
\text { status }\end{array}$ & $\begin{array}{c}\text { CCR+CPR } \\
\text { (cases) }\end{array}$ & $\begin{array}{c}\text { SD+PD } \\
(\text { cases })\end{array}$ & $\begin{array}{c}\text { CER } \\
(\%)\end{array}$ & P-value & pCR & P-value \\
\hline ER (+) & 111 & 51 & 73.0 & 0.001 & $10(6.7 \%)$ & 0.042 \\
ER (-) & 158 & 16 & 90.8 & & $16(9.2 \%)$ & \\
PR (+) & 98 & 44 & 69.0 & 0.001 & $8(5.6 \%)$ & 0.021 \\
PR (-) & 171 & 13 & 92.9 & & $18(9.8 \%)$ & 0.015 \\
HER-2 (+) & 135 & 19 & 87.7 & 0.028 & $17(11.0 \%)$ & \\
HER-2 (-) & 134 & 38 & 77.9 & & $9(5.2 \%)$ & \\
\hline
\end{tabular}

TE, docetaxel and epirubicin; TEC, docetaxel, epirubicin and cyclophosphamide; IP, intensive paclitaxel; CCR, clinical complete response; $\mathrm{CPR}$, clinical partial response; $\mathrm{SD}$, stable disease; PD, progressive disease; pCR, pathological complete response; CER, clinical effective rate; ER, estrogen receptor; PR, progesterone receptor; HER-2, human epidermal growth factor receptor 2.

Although current studies have shown that preoperative chemotherapy is not sufficient to improve the long-term survival rate of the overall population with breast cancer, results of a National Surgical Adjuvant Breast and Bowel Project (NSABP) B-18 large-scale randomized clinical study in 2001 manifested that the efficacy of preoperative chemotherapy is at least consistent with that of postoperative adjuvant chemotherapy, and many advanced patients who lose operation opportunity receive the operative treatment after preoperative chemotherapy, having significantly improved survival time (7). According to statistics, the overall clinical efficacy of NCT can be up to $60-90 \%$ currently, and 3\%-30\% patients can achieve pCR. At the same time, studies have revealed that patients who achieve pCR through NCT can obtain the ultimate survival advantage (8). pCR is currently one of the indexes of evaluating the efficacy of NCT or predicting the prognosis of patients.

It is recommended by the NCCN guideline and the St. Gallen expert consensus that the chemotherapy regimen containing paclitaxel and anthracycline is the first choice for NCT of breast cancer (9). At present, there is much application of double-combination or triple-combination regimens based on anthracycline and (or) paclitaxel in China, and the additional application of cytoxan can also obtain excellent efficacy in NCT (10). In the 1980s, Norton and Simon established a dose-dense model (11), based on which researchers further understood the tumor growth mode (12) and came up with a hypothesis that shortening the interval time of chemotherapy can kill the tumor cells more effectively. Therefore, paclitaxelintensive regimen was introduced into the clinical trials for the treatment of breast cancer. There are studies that pCR in paclitaxel-intensive chemotherapy group is higher, and both 5-year disease-free survival (DFS) and overall survival (OS) are also significantly higher than those in traditional 3-week chemotherapy group $(13,14)$. However, there are few studies on the efficacy of IP and the comparison with other chemotherapy regimens.

In this study, the clinical effective rate and pCR rate were compared among TE, TEC and IP groups. Results showed that there were no statistically significant differences in clinical effective rate and pCR rate among the three chemotherapy regimens, and no regimen with a better efficacy than other regimens was found. The difference in the incidence of neutropenia was statistically significant among the three groups. According to further analyses, the incidence rate of neutropenia was the highest in TEC group and the lowest in IP group. The severe bone marrow suppression is the most important cause of chemotherapy delay or dose reduction for breast cancer, which, if not detected or controlled in time, can lead to febrile neutropenia and secondary refractory infection, prolong the hospitalization time, increase treatment expense and even cause death. In this study, there was no difference in the clinical efficacy of TE, TEC and IP neoadjuvant chemotherapy, but the incidence rate of neutropenia in IP group was remarkably lower than those in TEC and TE groups. Therefore, IP can be considered in NCT for breast cancer patients at an advanced age or with poorer general conditions.

The efficacy of NCT is affected by a variety of factors, among which molecular typing can better reflect the biological behaviors of the tumor and the gene type in disease. Moreover, molecular typing is considered to be closely related to the pathological features, outcome, and prognosis and treatment response of tumors (15). Studies have demonstrated that the pCR rate is higher in triple-negative and HER-2-positive patients, but DFS and OS are lower with relatively poor prognosis (16-18). At present, there are few reports on the molecular typing of breast cancer and the efficacy of NCT. In this study, ER-negative, PR-negative and HER-2-positive states in the three groups were significantly correlated with the high clinical efficacy and high pCR rate, which were consistent with research results world-wide. The possible reason why the efficacy is affected by ER and PR is that the negative expression of ER and PR inhibits cell proliferation/growth and the expression of proto-oncogenes, and promote the expression of tumor suppressor genes, thereby regulating tumor progression in patients with breast cancer. In the clinical practice of NCT, appropriate population can be selected based on molecular typing. For example, NCT can be applied for HER-2-positive breast cancer patients or hormone receptor-positive patients with high expression of Ki-67.

There are many shortcomings in this study. The sample size was too small, so more clinical research objects should be enrolled for the large-sample data analysis to study the efficacy of different NCT regimens. In this study, only clinical efficacy, pCR rate and short-term efficacy were analyzed, 
patients were not followed up for a long time, and long-term survival and other relevant data were not collected, so whether there are differences in the long-term efficacy among the three regimens remains to be further explored and studied. It is hoped that multi-center clinical research on breast cancer NCT in the future can provide more accurate references for clinical decision and greater benefit for breast cancer patients.

In NCT for patients with stage II-III breast cancer, TE, TEC and IP neoadjuvant chemotherapy have similar efficacy. There are few adverse reactions and higher safety in IP group. ER-negative, PR-negative and HER-2-positive states are significantly correlated with the high clinical effective rate and high $\mathrm{pCR}$ rate.

\section{Acknowledgements}

Not applicable.

\section{Funding}

No funding was received.

\section{Availability of data and materials}

All data generated or analyzed during this study are included in this published article.

\section{Authors' contributions}

YL and FH designed the study and performed the experiments. ZX, ZZ and GW collected the data. YL and JS analyzed the data. YL and FH prepared the manuscript. All authors read and approved the final manuscript.

\section{Ethics approval and consent to participate}

This study was approved by the Ethics Committee of Shengjing Hospital of China Medical University (Shenyang, China). Signed informed consents were obtained from the patients or guardians.

\section{Patient consent for publication}

Not applicable.

\section{Competing interests}

The authors declare that they have no competing interests.

\section{References}

1. Rubovszky G and Horváth Z: Recent advances in the neoadjuvant treatment of breast cancer. J Breast Cancer 20: 119-131, 2017.

2. Gampenrieder SP, Rinnerthaler G and Greil R: Neoadjuvant chemotherapy and targeted therapy in breast cancer: Past, present, and future. J Oncol 2013: 732047, 2013.

3. Cain H, Macpherson IR, Beresford M, Pinder SE, Pong J and Dixon JM: Neoadjuvant therapy in early breast cancer: Treatment considerations and common debates in practice. Clin Onco (R Coll Radiol) 29: 642-652, 2017.
4. Omarini C, Guaitoli G, Pipitone S, Moscetti L, Cortesi L, Cascinu S and Piacentini F: Neoadjuvant treatments in triplenegative breast cancer patients: Where we are now and where we are going. Cancer Manag Res 10: 91-103, 2018.

5. Untch M, Konecny GE, Paepke S and von Minckwitz G: Current and future role of neoadjuvant therapy for breast cancer. Breast 23: 526-537, 2014.

6. Vila J, Teshome M, Tucker SL, Woodward WA, ChavezMacGregor M, Hunt KK and Mittendorf EA: Combining clinical and pathologic staging variables has prognostic value in predicting local-regional recurrence following neoadjuvant chemotherapy for breast cancer. Ann Surg 265: 574-580, 2017.

7. Hussain SA, Palmer DH, Stevens A, Spooner D, Poole CJ and Rea DW: Role of chemotherapy in breast cancer. Expert Rev Anticancer Ther 5: 1095-1110, 2005.

8. Cuppone F, Bria E, Carlini P, Milella M, Felici A, Sperduti I, Nisticò C, Terzoli E, Cognetti F and Giannarelli D: Taxanes as primary chemotherapy for early breast cancer: Meta-analysis of randomized trials. Cancer 113: 238-246, 2008.

9. Rastogi P, Anderson SJ, Bear HD, Geyer CE, Kahlenberg MS, Robidoux A, Margolese RG, Hoehn JL, Vogel VG, Dakhil SR, et al: Preoperative chemotherapy: Updates of National Surgical Adjuvant Breast and Bowel Project Protocols B-18 and B-27. J Clin Oncol 26: 778-785, 2008.

10. Margolin S, Bengtsson NO, Carlsson L, Edlund P, Hellstrøm M, Karlsson P, Lidbrink E, Linderholm B, Lindman H, Malmström P, et al; Scandinavian Breast Group Study SBG 2004-1: A randomised feasibility/phase II study (SBG 2004-1) with dose-dense/tailored epirubicin, cyclophoshamide (EC) followed by docetaxel ( $\mathrm{T}$ ) or fixed dosed dose-dense $\mathrm{EC} / \mathrm{T}$ versus $\mathrm{T}$, doxorubicin and $\mathrm{C}$ (TAC) in node-positive breast cancer. Acta Oncol 50: 35-41, 2011.

11. Norton L and Simon R: The Norton-Simon hypothesis revisited. Cancer Treat Rep 70: 163-169, 1986.

12. Norton L: A Gompertzian model of human breast cancer growth. Cancer Res 48: 7067-7071, 1988.

13. Untch M, Möbus V, Kuhn W, Muck BR, Thomssen C, Bauerfeind I, Harbeck N, Werner C, Lebeau A, Schneeweiss A, et al: Intensive dose-dense compared with conventionally scheduled preoperative chemotherapy for high-risk primary breast cancer. J Clin Oncol 27 : 2938-2945, 2009

14. Möbus V, Jackisch C, Lück HJ, du Bois A, Thomssen C, Kuhn W, Nitz U, Schneeweiss A, Huober J, Harbeck N, et al; AGO Breast Study Group (AGO-B): Ten-year results of intense dose-dense chemotherapy show superior survival compared with a conventional schedule in high-risk primary breast cancer: Final results of AGO phase III iddEPC trial. Ann Oncol 29: 178-185, 2018.

15. Houssami N, Macaskill P, von Minckwitz G, Marinovich ML and Mamounas E: Meta-analysis of the association of breast cancer subtype and pathologic complete response to neoadjuvant chemotherapy. Eur J Cancer 48: 3342-3354, 2012.

16. Carey LA, Dees EC, Sawyer L, Gatti L, Moore DT, Collichio F, Ollila DW, Sartor CI, Graham ML and Perou CM: The triple negative paradox: Primary tumor chemosensitivity of breast cancer subtypes. Clin Cancer Res 13: 2329-2334, 2007.

17. Chen XS, Wu JY, Huang O, Chen CM, Wu J, Lu JS, Shao ZM, Shen ZZ and Shen KW: Molecular subtype can predict the response and outcome of Chinese locally advanced breast cancer patients treated with preoperative therapy. Oncol Rep 23: 1213-1220, 2010.

18. Balko JM, Giltnane JM, Wang K, Schwarz LJ, Young CD, Cook RS, Owens P, Sanders ME, Kuba MG, Sánchez V, et al: Molecular profiling of the residual disease of triple-negative breast cancers after neoadjuvant chemotherapy identifies actionable therapeutic targets. Cancer Discov 4: 232-245, 2014.

This work is licensed under a Creative Commons Attribution-NonCommercial-NoDerivatives 4.0 International (CC BY-NC-ND 4.0) License. 